\title{
A Modified Cellular Automaton Model for Accounting for Traffic Behaviors during Signal Change Intervals
}

\author{
Chih-Cheng Hsu ${ }^{1}{ }^{1}$ and Yu-Chiun Chiou ${ }^{2}$ \\ ${ }^{1}$ Department of Traffic Management, Taiwan Police College, No. 153, Sec. 3, Singlong Rd., Wenshan District, Taipei 11696, Taiwan \\ ${ }^{2}$ Department of Transportation and Logistics Management, National Chiao Tung University, 4F, No. 118, Sec. 1, Chung Hsiao W. Rd., \\ Taipei 10044, Taiwan
}

Correspondence should be addressed to Chih-Cheng Hsu; chihcheng.hsu@gmail.com

Received 12 December 2017; Revised 18 March 2018; Accepted 25 March 2018; Published 9 May 2018

Academic Editor: Luigi Dell'Olio

Copyright (c) 2018 Chih-Cheng Hsu and Yu-Chiun Chiou. This is an open access article distributed under the Creative Commons Attribution License, which permits unrestricted use, distribution, and reproduction in any medium, provided the original work is properly cited.

\begin{abstract}
Previous cellular automata (CA) models have been developed for simulating driver behaviors in response to traffic signal control. However, driver behaviors during traffic signal change intervals, including cross/stop decision and speed adjustment, have not yet been studied. Based on this, this paper aims to propose a change interval CA model for replicating driver's perception and response to amber light based on stopping probability and speed adjusting functions. The proposed model has been validated by exemplified and field cases. To investigate the applicability of the proposed model, macroscopic and microscopic analyses are conducted. Although the macroscopic fundamental diagram analysis reveals only a small decrease in maximum traffic flow rates with considering driver behaviors in change intervals, in the microscopic analysis, the proposed model can present reasonable vehicular trajectories and deceleration rates during slowdown process.
\end{abstract}

\section{Introduction}

Modeling and simulation of urban traffic behaviors are essential to signal timing optimization and roadway design. Unlike highway traffic, urban traffic is inevitably disturbed by traffic signal at intersections and has closer interactions among neighboring vehicles. Obviously, it is much more challenging to model urban traffic behaviors than highway traffic behaviors. Cellular automata (CA) has been proved to be an effective approach to simulate urban traffic system; however, most of previous CA studies mainly focus on carfollowing and lane-changing behaviors accounting for major part of driving behaviors so as to optimize signal control strategy towards traffic efficiency or service capacity maximization accordingly (e.g., Brockfeld et al. [1], Gershenson and Rosenblueth [2], and Nagatani and Sugiyama [3]). In order to increase intersection safety, a signal change interval is to provide a safe transition between two conflicting traffic signal phases (e.g., McGee et al. [4], Gates et al. [5], and Zhang et al. [6]), which is common in most of signal timing plans.
Driver responses to the change interval differ remarkably to traditional car-following behaviors and are usually neglected in previous studies.

In the change interval, a driver at certain distance prior to the stop line of a signalized intersection may have an ambiguous decision to stop or cross the intersection. Such an ambiguous decision-making zone, namely, a dilemma zone, is essential to the signal timing design for traffic safety. However, the effect of signal change interval on traffic behaviors has been seldom considered in most vehicular traffic CA models. As driving along urban streets, drivers usually have to stop when they encounter a stationary traffic jam/obstacle or a red light. Most of CA models use car-following rules to replicate deceleration and stopping behaviors by correcting an abrupt speed change at the upstream front of a traffic jam or stationary obstacle with improvements to the limited deceleration capabilities or defensive reaction (e.g., Lan et al. [7], Lan and Chang [8], Lárraga and Alvarez-Icaza [9], and Neto et al. [10]). However, strictly speaking, the former is car-following behaviors interacting among drivers, while the 
latter is a decision-making behavior interacting among the driver, leading vehicles, and traffic lights, resulting into rather different vehicular trajectories and traffic patterns, especially, in the change interval. Based on this, the aim of this study is to propose and validate a modified CA model to replicate traffic behaviors during signal change interval.

The rest of this paper is organized as follows. The concept of generalized traffic parameters and the proposed forward CA rules are briefly explained in Section 2, wherein the updated rules of the change in yellow and red clearance intervals for vehicles and the logistics function for modeling drivers' decision of stopping are elucidated. In Section 3, the numerical simulation results for the proposed model are presented. The conclusions and suggestions for future studies are presented in Section 4.

\section{Models}

Lan et al. [7] proposed a refined CA model to avoid unrealistic abrupt deceleration when drivers encounter a red signal or traffic jam. However, since driver's response to a change interval may differ from a red signal or traffic jam, this paper modifies Lan's model to account for driving behaviors during a change interval. The three-dimensional generalized traffic variables, the Lan's model, and proposed modified model are discussed herein.

2.1. Generalized Traffic Variables. According to Lan et al. [7] and Lin et al. [11], the generalized spatiotemporal definitions of traffic variables, including occupancy $\rho(S)$, traffic flow $q(S)$, and space-mean speed $v(S)$ over a spatiotemporal domain $S$ can be defined as

$$
\begin{aligned}
& \rho(S)=\frac{\sum N_{0}(t) \Delta t}{\sum N \Delta t}=\frac{t(S)}{|S|}, \\
& q(S)=\frac{\sum M_{0}(x) \Delta x}{\sum T \Delta x}=\frac{d(S)}{|S|}, \\
& v(S)=\frac{q(S)}{\rho(S)}=\frac{d(S)}{t(S)} .
\end{aligned}
$$

In (1), the aforementioned traffic variables are used to depict the traffic flow patterns in the following CA simulations. Additionally, for clarity, Notations section summarizes all the variables and parameters used in this paper.

2.2. Forward Rules. To analyze driving behaviors with respect to a change interval, the forward rules, depending on the location of the vehicle, are divided into two parts. The first part indicates that a driver moves along a road segment. The second part represents the driver approaches to the signalized intersection. The steps of CA rules for two parts are narrated in detail as follows, respectively.

2.2.1. Moving along a Road Segment. The forward update rules that vehicles move along a road segment following the general CA forward rules proposed by Lan et al. [7], Jiang and $\mathrm{Wu}$ [12], and Knospe et al. [13] can be described as follows.
Step 1 (determination of randomization probability).

$$
P\left(v_{n}(t), t_{h}, t_{s}, S_{n+1}(t)\right)= \begin{cases}p_{b}: & \text { if } S_{n+1}=1, t_{h}<t_{s} \\ p_{0}: & \text { if } v_{n}=0, t_{\text {st }} \geq t_{k, c} \\ p_{d}: & \text { in all other cases }\end{cases}
$$

where $t_{h}=d_{n} / v_{n}(t), t_{s}=\min \left(v_{n}(t), h_{k}\right)$, and $t_{\text {st }}$ denotes the time at which the vehicle stops. The driver becomes less sensitive only when the car stops for more than a certain time $t_{k, c}$. The status identifier, $S_{n+1}(t)$, is determined in Step 5.

Step 2 (acceleration, determination of the speed of vehicles in the next time step).

$$
\begin{aligned}
\text { If } & \left(S_{n+1}(t)=0\right) \\
\text { or } \quad & \left(t_{h} \geq t_{s}\right) \\
\text { then } & v_{n}(t+1)=\min \left(v_{n}(t)+a_{k}, v_{k, \text { max }}\right) \\
\text { else } & v_{n}(t+1)=v_{n}(t),
\end{aligned}
$$

where $v_{k, \max }$ and $a_{k}$ are the maximum speed and acceleration capacity of a vehicle, respectively. Pure traffic is considered in this study; therefore, $k$ is a constant for a car.

Step 3 (deceleration). If $v_{n+1}(t+1)<v_{n}(t+1)$, the following safety criteria are considered to determine the speed at the next time step. Thus, drivers sense the relative speed to the vehicle in front and take precautionary reaction to prevent a collision only if the speed is positive relative to the front vehicle. In this study, the limited deceleration capability rules defined by Lan et al. [7] are further modified to apply to the intersection rules in the next subsection.

$$
\begin{gathered}
x_{n}(t+1)+\Delta+\sum_{i=1}^{\tau_{n}\left(c_{n}(t+1)\right)}\left(c_{n}(t+1)-D_{i}\right) \\
\leq x_{n+1}(t+1)+\sum_{i=1}^{\tau_{n}\left(c_{n}(t+1)\right)} v_{n+1}(t+1) .
\end{gathered}
$$

Step 4 (randomization).

$$
\begin{gathered}
\text { If } \quad(\operatorname{rand}()<p) \\
\text { then } \quad v_{n}(t+1)=\max \left(v_{n}(t+1)-1,0\right) .
\end{gathered}
$$

Step 5 (determination of vehicle status identifier $S_{n}(t)$ in the next time step).

$$
S_{n}(t+1)= \begin{cases}0 & \text { if } v_{n}(t+1)>v_{n}(t) \\ S_{n}(t) & \text { if } v_{n}(t+1)=v_{n}(t) \\ 1 & \text { if } v_{n}(t+1)<v_{n}(t) .\end{cases}
$$

Step 6 (determination of time $\left(t_{\mathrm{st}}\right)$ in a jam).

$$
t_{\mathrm{st}}= \begin{cases}t_{\mathrm{st}}=t_{\mathrm{st}}+1 & \text { if } v_{n}(t+1)=0 \\ t_{\mathrm{st}}=0 & \text { if } v_{n}(t+1)>0 .\end{cases}
$$


Step 7 (position update).

$$
x_{n}(t+1)=x_{n}(t)+\text { roundoff }\left(\frac{v_{n}(t)+v_{n}(t+1)}{2}\right) .
$$

2.2.2. Modifications for the Behaviors during Change Interval. The duration of change intervals included amber warning indication and red clearance indication. The amber indication tells an approaching driver that the right of way is about to be assigned to a conflicting traffic flow, while, the red clearance indication is provided to allow vehicles in the intersection to clear before the green is given to conflicting traffic. During the period of time, drivers may decide to stop or to cross the intersection depending on how far to the intersection and how fast the driving speed.

To account for the driver behavior in change intervals, the modified CA model (hereafter referred to as MCA) mainly included a driver's perception and response to the amber and red lights and the corresponding deceleration process as approaching to a signalized intersection. Behaviors of drivers encountering amber and red intervals are formulated by following six-step rules from (9) to (21) subsequently.

Step 8 (orientation). The initial time step is used to examine the number of vehicles encountering a traffic light within a default activation region, $d^{\text {ac }}$, where drivers are influenced by the amber indication, as expressed in (9). The other vehicles followed their leading vehicles and the forward rules for reaction irrespective of the color of the traffic light, as expressed in (10).

$$
\begin{array}{ll}
\text { for } & i=0, \\
\text { if } & d_{n}^{\mathrm{sig}}(t+1)=x^{\mathrm{sig}}-x_{n}(t) \leq d^{\mathrm{act}},
\end{array}
$$

then Go to Step 2 Eq. (11)

$$
\text { else } \begin{aligned}
& d_{n}(t+1) \\
&= x_{n+1}(t) \\
&-x_{n}(t) \text { and go to the Forward Rules. }
\end{aligned}
$$

Step 9 (perception). The vehicles entering the activation region are set as the subject vehicles, as expressed in (11). The leading subject vehicle closest to the intersection $\left(\operatorname{Sub}_{n}(t)=\right.$ 1 ) is considered the last-to-cross or first-to-stop vehicle at the end of change intervals. The last-to-cross subject vehicle follows its leading vehicle to cross the intersection, and the first-to-stop subject vehicle guides the following vehicles to stop.

$$
\operatorname{Sub}_{n}(t+1)= \begin{cases}1 & \text { if the road sites from } x_{n}(t) \text { to } x^{\text {sig }} \text { are not occupied by other vehicles } \\ 0 & \text { in all other cases. }\end{cases}
$$

\section{Step 10 (reaction).}

Step 3.1 (status determination). A new vehicle's status identifier, $S_{n}^{a}$, is added to describe a driver's decision to stop or to cross at the onset of the amber light. As shown in (12), the identifier is based on two conditions. The first condition is to introduce a distance-dependent stopping probability function to reflect the uncertainty of a driver's decision: as the distance to the intersection decreases, the stopping probability of a driver also decreases. The stopping probability is formulated from the logistic function discussed in next section. The second condition is to consider the influence of the preceding vehicle. For a leading subject vehicle, $\operatorname{Sub}_{n}(t)=1$, the identifier is determined by the first condition only. However, the identifier of the other subject vehicles, $\operatorname{Sub}_{n}(t)=0$, is determined by the identifier of the preceding vehicle, implying that the subject vehicle must stop when its leading vehicle has a "stop status" and the stop probability function is used to determine the identifier status of the subject vehicle when its leading vehicle presents a "cross status." Accordingly, all the subject vehicles can be divided into two statuses, "stop status" $\left(S_{n}^{a}=1\right)$ and "cross status" $\left(S_{n}^{a}=0\right)$.

$$
\text { if } \begin{aligned}
& \operatorname{Sub}_{n}(t)=1 \\
& \operatorname{rand}()>P_{a}
\end{aligned}
$$

$$
\begin{aligned}
\text { then } & S_{n}^{a}(t+1)=1 \\
\text { else } & S_{n}^{a}(t+1)=0 \\
\text { if } & S_{n}(t)=0, \\
& S_{n+1}^{a}(t+1)=1 \\
\text { then } & S_{n}^{a}(t+1)=1 \\
\text { else } & \operatorname{rand}()>P_{a} \\
\text { then } & S_{n}^{a}(t+1)=1 \\
\text { else } & S_{n}^{a}(t+1)=0
\end{aligned}
$$

The logistic function is one of the most commonly used research methods to determine the stopping probability, which describes the relationship between the stopping probability of a vehicle and its distance to intersection at the onset of an amber light. The logistic function is a graph of an Sshaped curve, as expressed in (13), where $x$ is the explanatory variable and $\alpha, \beta$, and $\gamma$ are relevant parameters shaping the curve. As shown in Figure 1, $\alpha$ is associated with the steepness of the curve, $\beta$ is the $x$-value of the curve's midpoint, and $\gamma$ determines the maximum value of the curve, respectively. The logistic function is incorporated in the proposed MCA 

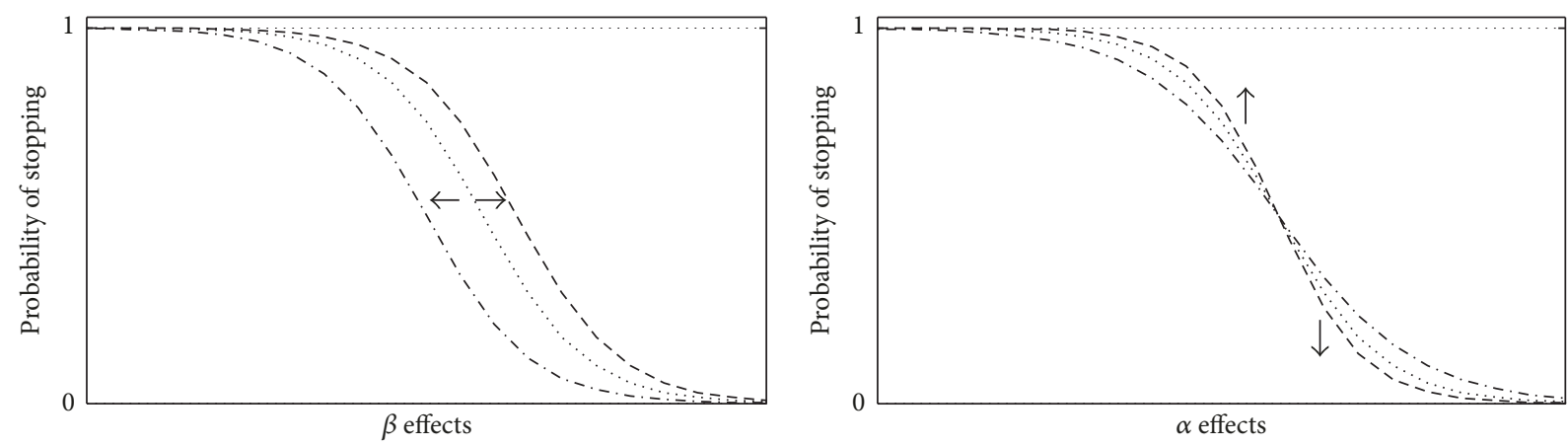

FIGURE 1: Shape changes in the logistic curves under various parameter settings.

model to elucidate drivers' decision uncertainty of whether to stop or cross under change interval. As expressed in (14), the new random term, $P_{a}$, that is the normal form of the logistic function in (13) $(\gamma=1)$, is proposed. The stopping probability of the driver depends on the variable, $x$, which is the distance between the vehicle and the intersection. Furthermore, (14) is divided by $1-P_{a}$, and a natural logarithm is taken on both sides; then, the function can be converted into a linear model, as expressed in (15).

$$
\begin{aligned}
& f(x ; \gamma, \alpha, \beta)=\frac{\gamma}{1+e^{-\alpha(x-\beta)}}, \\
& P_{a}\left(d_{n}^{\text {sig }} ; \alpha, \beta\right)=\frac{1}{1+e^{-\alpha\left(d_{n}^{\text {sig }}-\beta\right)}}, \\
& \ln \left(\frac{P_{a}\left(d_{n}^{\text {sig }} ; \alpha, \beta\right)}{1-P_{a}\left(d_{n}^{\text {sig }} ; \alpha, \beta\right)}\right)=\ln \left(\frac{1}{e^{-\alpha\left(d_{n}^{\text {sig }}-\beta\right)}}\right) \\
& \quad=-\alpha \beta+\alpha d_{n}^{\text {sig }} .
\end{aligned}
$$

Step 3.2 (unfulfilled conditions exclusion). Two excluded conditions related to vehicle's status derived from Step 3.1 are modified as explained herein.

Step 3.2.1 (cannot-stop condition). A subject vehicle with a stop status is to decelerate from the current velocity with default maximum deceleration rate at each time step, and if the vehicle cannot stop prior to the intersection in the remaining amber light duration, the condition is defined as the "cannot-stop condition"; the vehicle has a cross status, implying the vehicle is assigned to follow its leading vehicle by the forward rules in the Lan's model and skip the change interval rules, as expressed in (16). Additionally, the deceleration function of the vehicles with stop status is described in (20).

$$
\text { if } \begin{aligned}
& S_{n}^{a}(t+1)=1, \\
& x_{n}(t)+\sum_{i=1}^{\tau^{a}-1}\left(\max \left(v_{n}(t+1)-D, 0\right)\right)>x^{\mathrm{sig}}
\end{aligned}
$$

then $S_{n}^{a}(t+1)=0$ and Go to the Eq. (10)

else Go to the Eq. (20)
Step 3.2.2 (cannot-cross condition). If a subject vehicle driver decides to cross but is not able to reach the intersection with the default acceleration rate, the vehicle's identifier is altered to stop status, as expressed in (17). That is, the vehicle "cannotcross condition" is used to slow down the vehicle at the stop line for the upcoming red light according to its current speed and distance of its location to the intersection, as expressed in (20). The subject vehicles excluded are updated with the forward rules, as expressed in (10).

$$
\text { if } \begin{aligned}
& S_{n}^{a}(t+1)=0 \\
& x_{n}(t)+\sum_{i=1}^{\tau^{a}-1}\left(\min \left(v_{n}(t+1)+a, 0\right)\right)<x^{\text {sig }}
\end{aligned}
$$

then $S_{n}^{a}(t+1)=1$ and Go to the Eq. (20)

else Go to the Eq. (10)

Step 11 (maintaining speed). Steps 2 and 3 present driver's perception and reaction procedures for encountering amber light onset. The driver must recognize the amber indication and decide to stop or to cross when approaching the intersection at the beginning of the change interval. The perception and reaction time in this process may vary individually. For simplification, a constant value of one second, suggested by McGee et al. [4], is adopted. Drivers are supposed to maintain a constant speed in this step, as expressed in the following:

$$
\begin{aligned}
\text { if } & \operatorname{Sub}_{n}(t+1)=1 \text { or } 0 \\
\text { then } & v_{n}(t+1)=v_{n}(t) .
\end{aligned}
$$

Step 12 (amber light interval forward rules). The subsequent rules are defined for a vehicle with stop status to slow down at the intersection in the remaining duration of the amber light interval. A velocity adjusting function modified from Lan's limited deceleration function [7] is proposed which is referred to as an ideal situation for the driver of a vehicle with "stop status." It is assumed that drivers tend to slow down their vehicle to stop at the red light with a comfortable deceleration rate at each time step, as expressed in (19). The deceleration rate is predetermined according 
to ITE Traffic Engineering Handbook [14]. Based on this assumption, a vehicle is set to decelerate from the maximum speed to stop at a fixed deceleration rate per time step. The vehicle's speed and its corresponding braking distance at each time step are considered the criterion speed and braking distance. Considering these criterion values, the comfortable deceleration process can be divided into several speed-space intervals. In (20), the $n$th vehicle with stop status is considered as an example. In a speed-space interval, criterion speed $c^{*}$ and criterion distance $d^{*}$ were the lower bounds, and $c^{u}$ and $d^{u}$ were the upper bounds. If the subject vehicle's speed is within $\left[c^{u}, c^{*}\right]$ and distance to the intersection is within $\left[d^{*}, d^{u}\right]$ indicating the vehicle is situated at the criterion range, the comfortable deceleration rate $D_{c}$ is applied at this time step. If the distance to the intersection is less than $d^{*}$, the driver cannot comfortably slow down to stop and the maximum deceleration rate $D$ is employed. In contrast, if the distance to the intersection is more than or equal to $d^{u}$, the vehicle is set to a constant velocity to be ready to stop at the red light.

$$
\begin{aligned}
& x_{n}(t)+\left(v_{n}(t+1)-1 * D_{c}\right)+\left(v_{n}(t+1)-2 * D_{c}\right)+\cdots+\max \left(\left(v_{n}(t+1)-\tau * D_{c}\right), 0\right) \leq x^{\text {sig }} \\
& \text { for } i=1 \text {; } \\
& i \leq \tau^{a}-1 \\
& i++ \\
& \text { If } c^{*}(t+1) \leq v_{n}(t+1)<c^{u}(t+1) \\
& \text { then } \quad v_{n}(t+1)= \begin{cases}v_{n}(t+1)-D & \text { if } d_{n}^{\text {sig }}(t+1)<d^{*}\left(c^{*}(t+1)\right) \\
v_{n}(t+1)-D_{c} & \text { if } d^{*}\left(c^{*}(t+1)\right) \leq d_{n}^{\text {sig }}(t+1)<d^{u}\left(c^{u}(t+1)\right) \\
v_{n}(t+1) & \text { if } d_{n}^{\text {sig }}(t+1) \geq d^{u}\left(c^{u}(t+1)\right) .\end{cases}
\end{aligned}
$$

Step 13 (red-light interval forward rules). At a red light, the criterion stop rule continues to be applied to the driver as expressed in (20), except for two conditions. Firstly, if drivers have already entered the intersection at the first time step of the red light, their decision is to continue to cross the intersection in the rest of the red-light duration, as expressed in (21). A permissive law, indicating if a vehicle with cross status is supposed to cross the intersection certainly in the yellow and all-red clearance intervals, is adopted. Secondly, if a vehicle's speed is less than or equal to 5 sites/time step, the conventional NaSch deceleration rule [15] is adopted to brake the vehicle properly without exceeding deceleration capability.

$$
\begin{array}{ll}
\text { for } \quad & i=\tau^{a} \\
& i \leq \tau^{a}+\tau^{r}-1 \\
& i++ \\
\text { if } \quad i=\tau^{a}, x_{n}(t)+v_{n}(t+1)>x^{\mathrm{sig}}
\end{array}
$$

then Go to the Eq. (10)

else if $v_{n}(t+1) \leq 5$

then $v_{n}(t+1)=\min \left(d_{n}^{\text {sig }}(t+1), v_{n}(t+1)\right)$

else Go to the Eq. (20).

\section{Results}

This section is organized by three subsections: an exemplified case for validation, the crossing and stopping boundaries, and microscopic and macroscopic analyses. The scenarios are set to be identical to those of Lan et al. [16]; that is, the simulations are performed on a closed track containing $6 \times$ 1800 site CUs, representing a two-lane roadway section of width $7.5 \mathrm{~m}$ and length $1800 \mathrm{~m}$, and a traffic signal is placed at the middle of the road. Pure traffic scenario (cars only) was simulated. Initially, all the vehicles were set lined up from the end of the road section on the circular track, with speed 0 at time step 0 . The maximum speed for cars was set according to the speed limits $(50 \mathrm{~km} / \mathrm{h})$ prevailing in Taiwan urban streets, that is, 14 sites/s. The signal cycle time was $90 \mathrm{~s}$, including the red interval of $30 \mathrm{~s}$, change interval containing amber light duration of $3 \mathrm{~s}$ ( or $5 \mathrm{~s}$ ), an all-red duration of $2 \mathrm{~s}$, and a green interval of $55 \mathrm{~s}$ (53 s). Three-level speed scenarios for a crossing boundary-stopping boundary (CB-SB) zone setting, a low speed of 6 sites/s, medium speed of 10 sites/s, and a high speed of 13 sites/s are considered. On the basis of previous studies $[4,16,17]$, the maximum and comfortable deceleration rate were 5 and $3 \mathrm{~m} / \mathrm{s}^{2}$, whereas the maximum acceleration rate was set as $4 \mathrm{~m} / \mathrm{s}^{2}$. The other parameters were set as $P_{b}=0.94, P_{0}=0.5, P_{d}=0.1, t_{c}=6$, and $h=4$. The logistic function parameter $\gamma=1, \alpha=0.17$, and $\beta=-33,-27,-20$, and $-25 \mathrm{~m}$ for high speed, medium speed, low speed, and allvehicle scenarios, respectively. 


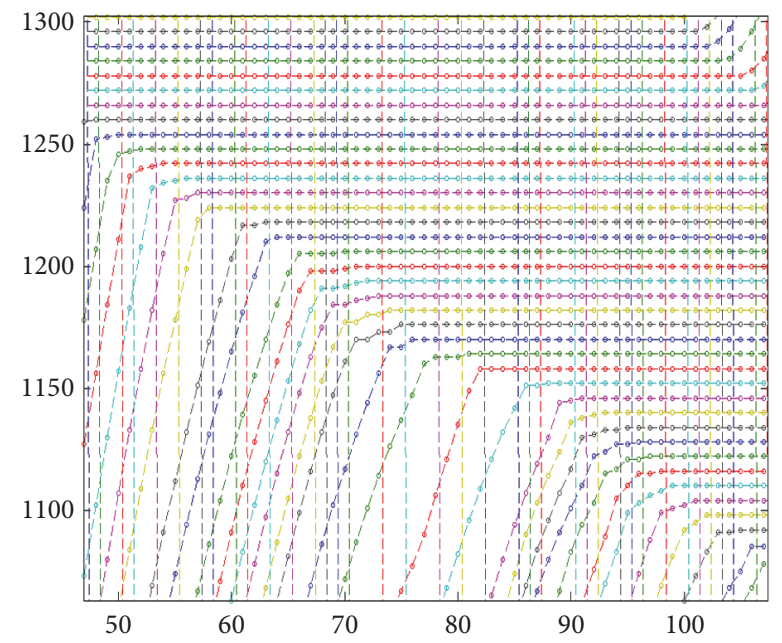

(a) Hsu et al's model

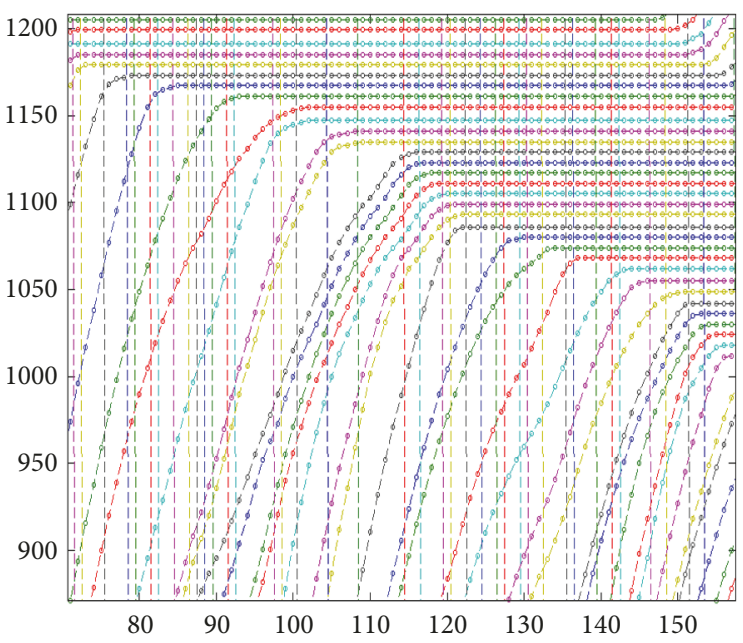

(b) Lan et al's model

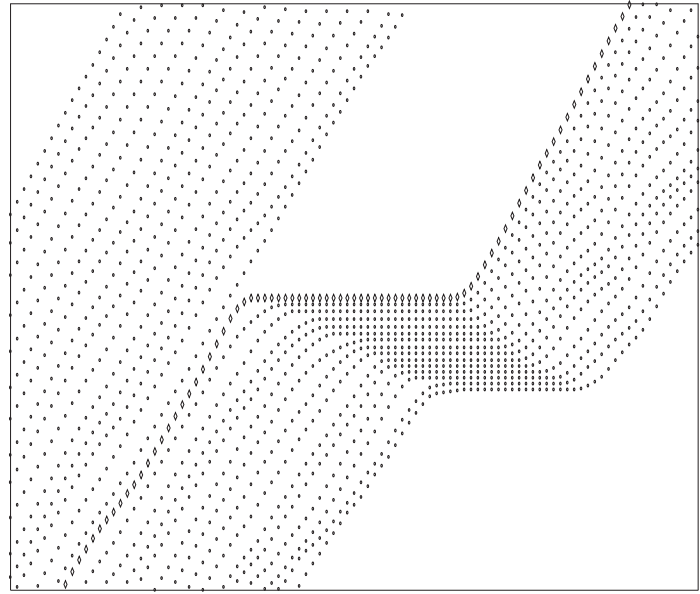

(c) The present model

FIGURE 2: Vehicular trajectories approaching the upstream front of a traffic jam or traffic light in the yellow-light and red-light change intervals.

3.1. Exemplified Case for Validation. The proposed MCA model was examined by the following exemplified case. Different maximum velocities are set for the vehicles approaching to the intersection. Two criteria were selected for validating this model: (a) check the reasonability of the transition of the vehicular trajectories, as shown in distance time $(x-t)$ diagrams; (b) check the reasonability of vehicles' velocities at each time step during deceleration process.

The deceleration process and stopping decision of a driver in response to a change interval or red light are different from the condition encountering upstream front of a traffic jam, since the driver does not act as car-following behavior but interacts with traffic lights. A driver reacting to the yellowlight onset will have a time lag of perception and response time prior to slowing down the vehicle. Figures 2 and 3 illustrate the trajectories of vehicles' deceleration process. Moreover, according to the deceleration rules established, the deceleration rate must not exceed the maximum deceleration rate. Figure 2 compares the simulated $x$-t diagrams with two previously proposed CA models $[7,18]$. The horizontal and vertical axes are time (s) and locations (m) of vehicles. The time space diagram indicates that vehicles encountered congestion and decelerated to stop, as shown in Figure 2(a), as proposed by $\mathrm{Hsu}$ et al. [18]. A sharp speed drop for a sudden stop when vehicles encountered traffic congestion was observed. To overcome this problem, Lan et al. [7] proposed a limit deceleration method in which a vehicle is set to maintain a safe travel speed behind the leading vehicle at any time step when its velocity is higher than the vehicle in front. Figure 2(b) shows arc-shaped vehicle trajectories, indicating that the vehicles decelerated in advance before approaching the upstream front of the traffic jam. As shown in Figure 2(c), the trajectory presents a smooth curve from the onset of yellow light to a complete stop. The subject vehicle does not show the aforementioned behavior of a sudden stop and it also does not decelerate in advance. Figure (3) describes the behaviors in response to change interval in more detail.

Figures 3(a), 3(c), and 3(e) show the simulated $x-t$ diagrams with high-, medium-, and low-speed scenarios. The trajectory of the subject vehicle is highlighted by the bold 


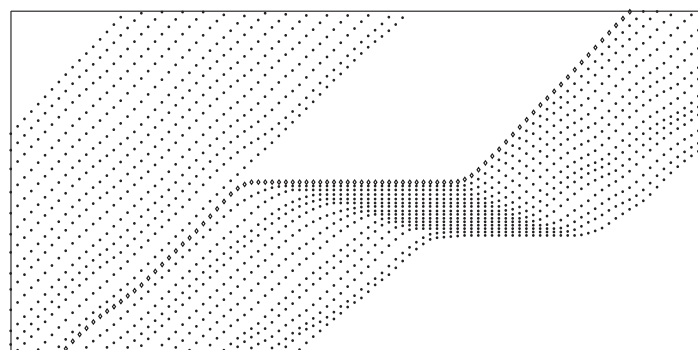

(a) $x$ - $t$ diagram of the high-speed scenario

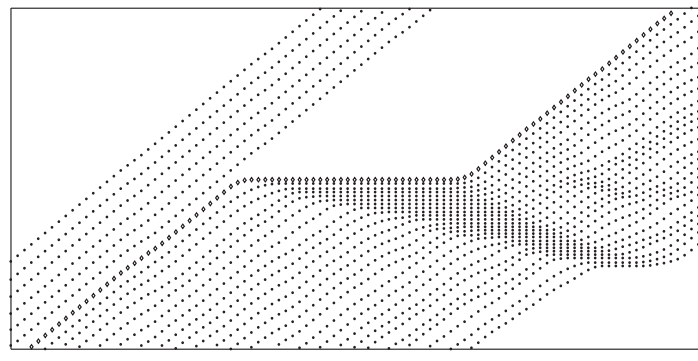

(c) $x$ - $t$ diagram of the medium-speed scenario

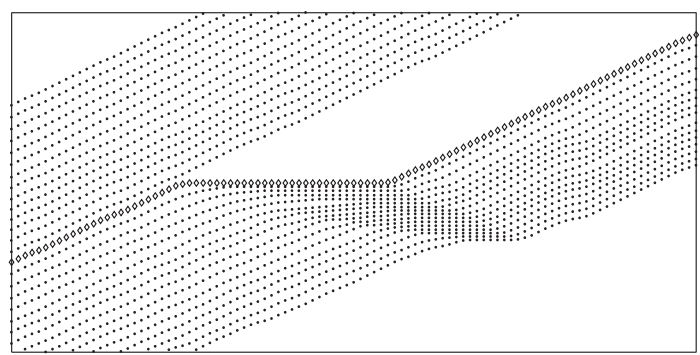

(e) $x-t$ diagram of the low-speed scenario

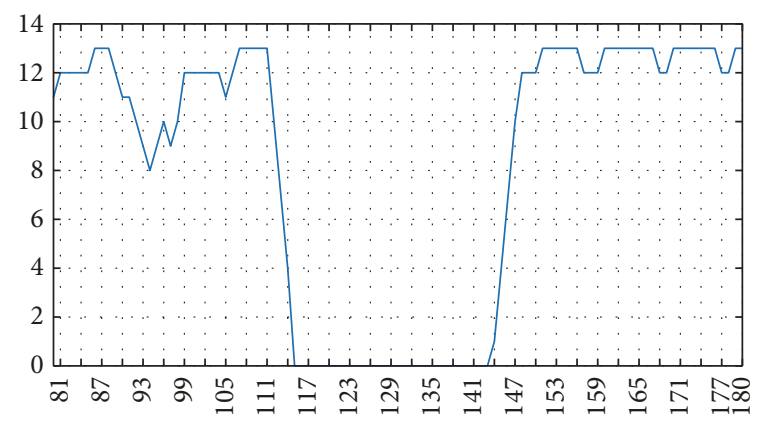

(b) $v$ - $t$ diagram of the high-speed scenario

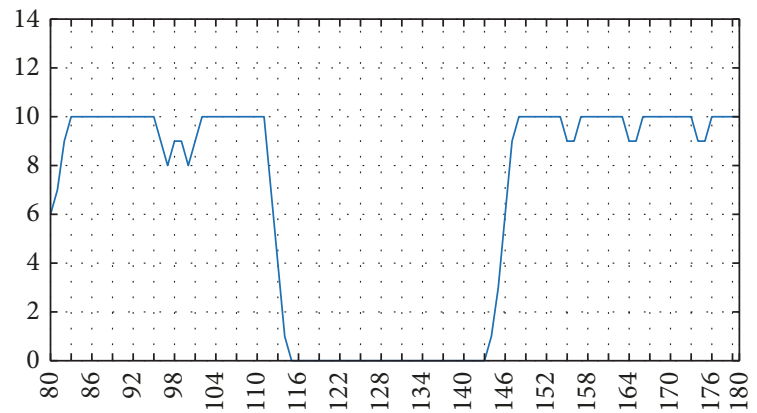

(d) $v$ - $t$ diagram of the medium-speed scenario

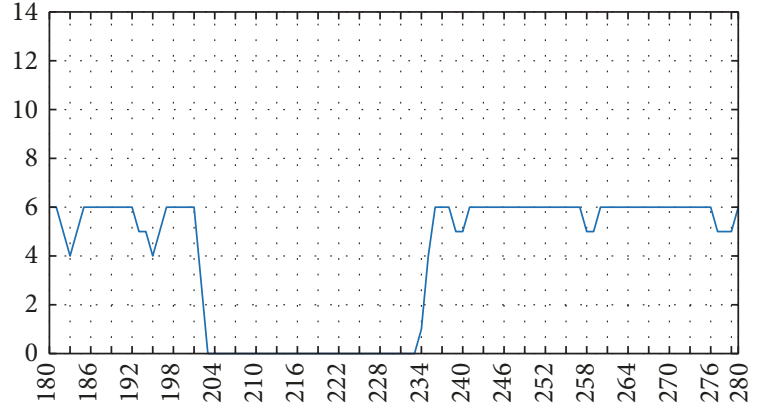

(f) $v$ - $t$ diagram of the low-speed scenario

FIGURE 3: Vehicular trajectories and deceleration rates under various speed conditions when approaching the intersection during the change interval.

dotted lines in Figures 3(a), 3(c), and 3(e). The vehicular trajectories are similar to each other in the deceleration process even if the vehicles approach the intersection with various speeds. Figures 3(b), 3(d), and 3(f) represent the simulated $v$ - $t$ diagrams. A vehicle's complete stop time does not depend on the red-light onset but is determined by the deceleration rates of the rules. As the deceleration rate is applied to the vehicle in these cases, the minimum duration of the deceleration process is the low-speed scenario where the subject vehicle with $1 \mathrm{~s}$ time maintains a constant speed, with a deceleration rate of $3 \mathrm{~m} / \mathrm{s}$ for $2 \mathrm{~s}$ to stop, as shown in Figure 3(f). The durations of the high-speed and mediumspeed scenarios are $3 \mathrm{~s}$ and $4 \mathrm{~s}$, respectively, from deceleration to stop. In the medium-speed scenario, the vehicle with $1 \mathrm{~s}$ maintains constant speed, with a deceleration rate of $3 \mathrm{~m} / \mathrm{s}$ for $2 \mathrm{~s}$ and $1 \mathrm{~m} / \mathrm{s}$ for $1 \mathrm{~s}$ to stop, as shown in Figure 3(d). In the high-speed scenario, the vehicle with $1 \mathrm{~s}$ time maintains a constant speed and a deceleration rate of $3 \mathrm{~m} / \mathrm{s}$ for $3 \mathrm{~s}$ and $4 \mathrm{~m} / \mathrm{s}$ for $1 \mathrm{~s}$ to stop, as shown in Figure 3(b). The vehicular trajectories and variations in vehicle speed at each time step are reasonable during the deceleration processes. The startup delay in a signalized intersection, as shown in Figures 3(b), 3(d), and 3(f), will be discussed in subsequent research and is thus not discussed here.

3.2. Crossing and Stopping Boundaries. Figure 4 illustrates what the MCA model is expected to present under the aforementioned parameter settings. Firstly, the farthest distance at which a vehicle can cross the intersection within the change intervals is defined as the "crossing boundary" (CB). Vehicles beyond the CB are considered to have a "cannotcross condition." In contrast, the nearest distance at which a vehicle can stop at the red light is defined as the "stopping boundary" (SB). Vehicles within the SB are considered to have a "cannot-stop condition." Three CB-SB zones are formed by the simulations of three levels of travel speed. In Figure 4, the vertical blue solid lines represent the CB-SB zone of the high-speed scenario. The location of the left vertical blue line, 


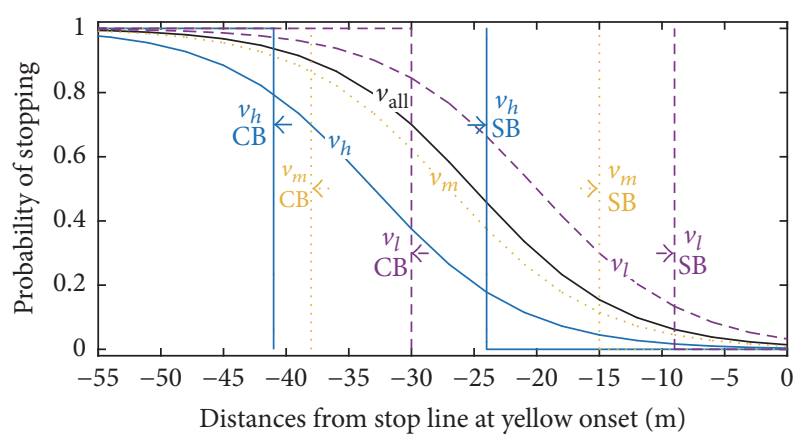

FIgURE 4: Logistic function curves and CB-SB zones shift under different velocities.

$\mathrm{CB}$, indicates the boundary position that a vehicle can cross. On the left side of this position, the stopping probability of the vehicle is 1 . Similarly, the location of the right vertical blue line, SB, represents the boundary position that a vehicle can stop. On the right side of this position, the stopping probability of the vehicle is 0 . The $\mathrm{CB}-\mathrm{SB}$ zones of the medium-speed and low-speed scenarios are represented by the yellow dotted line and the purple dashed line in Figure 4. The position of the $\mathrm{CB}$ line represents a crisp value indicating the driver's binary decision whether to cross or to stop the intersection, similar to the position of the SB line.

However, the stopping probability of a vehicle decreases gradually as the vehicle approaches the intersection. Therefore, the logistic function is used to depict the relationship between a driver decision under change interval uncertainty and the distance to the intersection. Firstly, three logistic function curves associating with high-, medium-, and lowspeed scenarios are established in Figure 4. In (15), the logistic function becomes a linear function once the distance, $d_{n}^{\text {sig }}$, is an explanatory variable. In this model, the arithmetic mean of two distances of $\mathrm{CB}$ and $\mathrm{SB}$ is set as $d_{n}^{\text {sig }}$. As the vehicle speed decreases from high to low, $d_{n}^{\text {sig }}$ also decreases, resulting in the $\mathrm{CB}-\mathrm{SB}$ zones and logistic curves moving toward the intersection. Furthermore, Figure 4 presents the deterministic results of the MCA model.

\subsection{Microscopic and Macroscopic Analyses}

3.3.1. Macroscopic Analysis. To investigate how much traffic flow is affected by the change interval, fundamental diagrams of three scenarios with various yellow-light durations ranging between 0,3 , and $5 \mathrm{~s}$ are compared. As shown in Figure 5, three global flow-occupancy curves are considerably close. That is, the increase of change interval duration only slightly affects the global traffic flow rate. On the basis of the $3 \mathrm{~s}$ amber light time case, to increase the yellow-light time by $2 \mathrm{~s}$ or decrease by $3 \mathrm{~s}$, the total number of vehicles passing through the intersection may be different by only 1 to 2 vehicles. If amber- and all-red intervals are both removed, the maximum flow rate can increase up to $18 \%$ as shown in Figure 6. However, the signalized intersection without change interval will result in lots of potential traffic conflicts and even accidents.

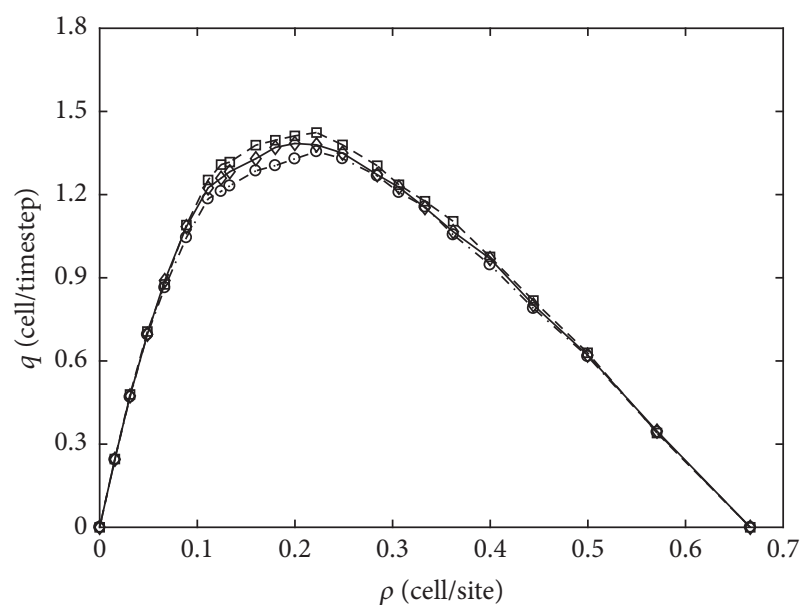

- - Amber-light interval $0 \mathrm{~s}$

$\rightarrow$ Amber-light interval $3 \mathrm{~s}$

$-0-$ Amber-light interval $5 \mathrm{~s}$

FIgURE 5: Fundamental diagrams under different change interval durations.

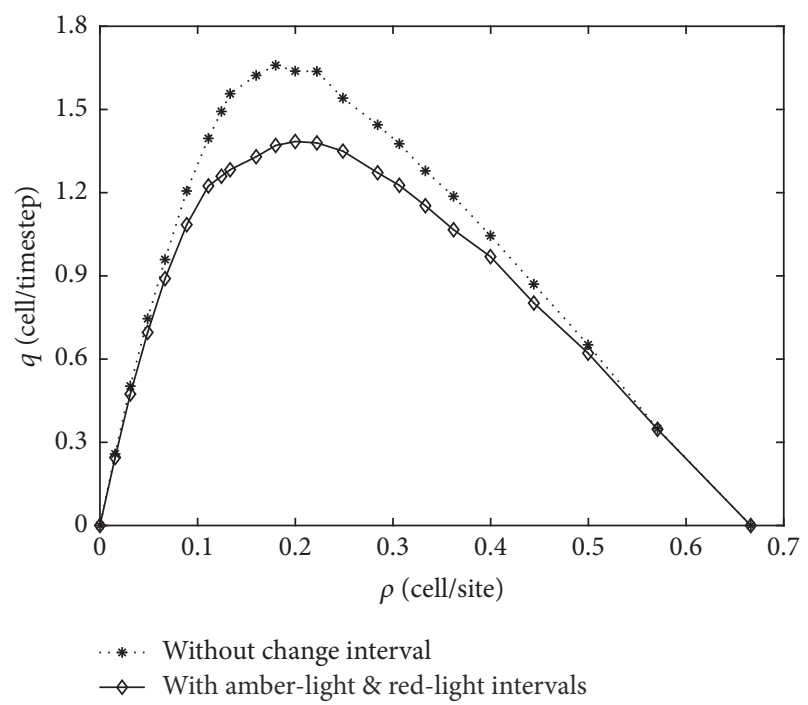

FIGURE 6: Fundamental diagrams with and without change interval.

3.3.2. Microscopic Analysis. To take a microscopic view of drivers' behaviors during change intervals, Figure 7 presents the time space diagram of vehicular trajectories during change intervals. As shown in Figure 7, the vehicles approaching the intersection within the activation distance at the amber light onset are considered as the subject vehicles. The closest vehicle from the intersection with the diamond mark is the last-to-cross vehicle $\left(\operatorname{Sub}_{n}=1\right)$, and circle mark represents the first-to-stop vehicle $\left(\mathrm{Sub}_{n}=0\right)$. Because the vehicle $\left(\operatorname{Sub}_{n}=1\right)$ in Figure 7 reaches the intersection after the perception and response time, it passes directly without being affected by traffic light change. In contrast, the driver of the following vehicle $\left(\mathrm{Sub}_{n}=0\right)$ perceives the yellow onset and decides to stop. Figure 7 clearly depicts the vehicle trajectory 


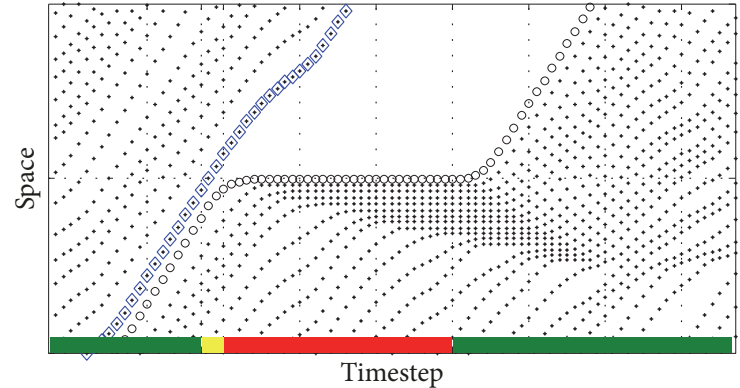

FIGURE 7: $x$ - $t$ plots of vehicular trajectories during the change intervals.

during the deceleration process between the yellow-light and red-light intervals.

To analyze the stopping probability of a vehicle in the change interval, the data derived from numerical simulations under various initial occupancies are used. The relevant information of the subject vehicle includes the distance of the vehicle from the intersection, travel speed at yellow onset, and stopping or crossing. This information was extracted from the samples of the following analysis. The full dataset includes 594 vehicles, which can be roughly divided into two categories, to stop and to cross the intersection. In Figure 8(b), the shape of the distribution of the vehicles to cross is left skewed, while, in contrast, the shape of the distribution of to stop vehicles is right skewed. An overlapping zone is also observed in the middle. Therefore, the zone approaching the intersection can be roughly divided into three categories, to stop, to stop-orcross, and to cross, respectively.

The distribution of the samples is visualised in a velocityspace scatter plot, as shown in Figure 8(a). As noted from Figure 8, at the distance less than $15 \mathrm{~m}$ to the intersection, most of vehicles no matter what speed they travel tend to cross the intersection, while at the distance larger than $40 \mathrm{~m}$, most of vehicles tend to stop. At the distance between 15 and $40 \mathrm{~m}$, the proportion of vehicles to cross increases as travel speed is higher. The dilemma zone, a well-known effect of change interval, can be easily identified from Figure 8 .

Based on the scatter plot of the samples, the vehicles' stopping probability during the change interval is shown in Figure 9, where the $x$-axis represents the positions of vehicles approaching to the intersection at three levels of velocities and the $y$-axis represents the stopping probability. The aforementioned samples are used to form the curve of the stopping probability as shown in Figure 9(a). As the distance from the intersection increases/decreases, the probability of stopping also increases/decreases. In Figure 9(a), when vehicles are $40 \mathrm{~m}$ from the intersection, the crossing probability becomes zero. As the distance decreases, the stopping probability becomes rapidly decreasing until the midpoint, which is an inflection point at a position of $25 \mathrm{~m}$. Thereafter, the probability value continues to decrease, but the rate of decrease slows down until a position of $10 \mathrm{~m}$. Finally, the stopping probability approaches to zero as the distance is less than $10 \mathrm{~m}$. The curve is further compared with the logistic function curve. The entire curve has a good fit with

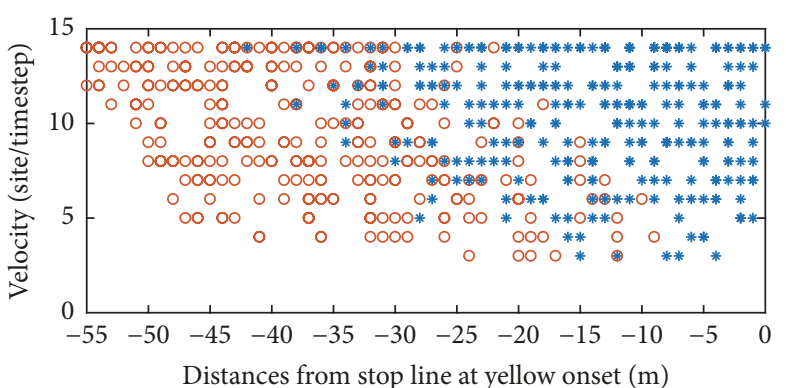

* Crossing

○ Stopping

(a)

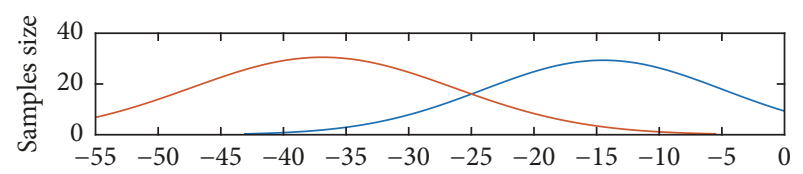

(b)

FIgURE 8: Probability of a vehicle stopping or crossing at different velocities related to the distance from the intersection at yellow-light onset.

the logistic function curve, especially in the range of $25-40 \mathrm{~m}$, as outside the range, there are few cases in which the MCA rules for to stop or to cross are different from what the logistic curve indicated. That is, the probability value $P_{a}$ generated by the logistic function may indicate the vehicle to stop/to cross, but the status of the vehicle is cannot-stop/cannotcross because it matches the "unfulfilled condition exclusion" rules. For further inspection of the CB-SB zone boundary depicted by the model, at the onset of the yellow light, the vehicle's distance to the intersection is between 10 and $40 \mathrm{~m}$, which corresponds to the $5 \%$ to $93 \%$ stopping region. In Figure 9(b), the samples are divided into three vehicle speed scenarios for further analysis. The simulated curves represent drivers with different approaching velocities and demonstrate a pattern similar to the stopping probability. The shift of the curves to the right indicates that, as drivers approach the intersection, the low-speed drivers stop at a shorter distance from the intersections than the high-speed drivers under a fixed probability. In contrast, the shift of the curves to the left indicates that the high-speed drivers decide to cross at a longer distance from the intersection than the low-speed drivers, as shown in Figure 9(b). Comparing the curves formed by three speed levels to those formed by the samples, it can be observed that drivers' speeds have a clear effect on the distances of their decisions to stop or to cross when they encounter change intervals. With an increase in variation in vehicle speeds, the dilemma zone enlarges accordingly.

3.4. Field Case for Validation. The simulated stopping probability curve is further compared with real traffic data collected by Institute of Transportation, Ministry of Transportation and Communications, Taiwan [19]. A total of 1,199 vehicles sampled from 13 signalized intersections in north and central Taiwan are selected. The dataset contains information 


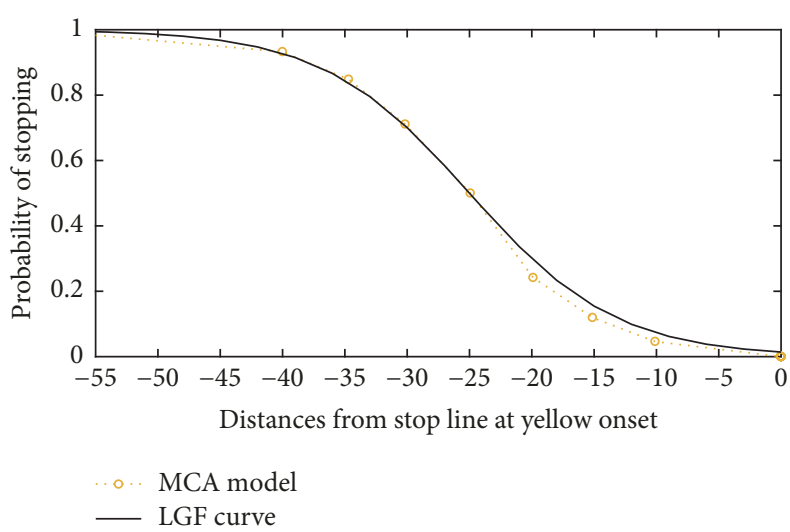

(a) Simulated curve versus logistic curve

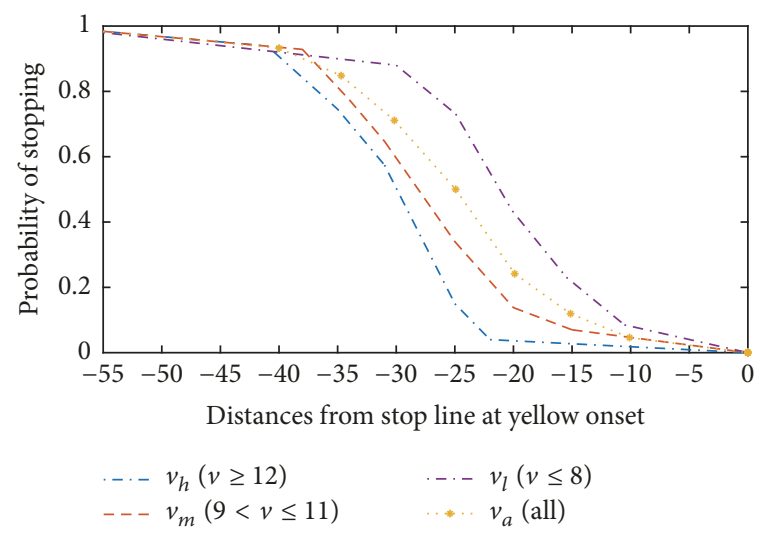

(b) Simulated curves under various velocities

FIGURE 9: Stopping probability at various distances and velocities at yellow-light onset.

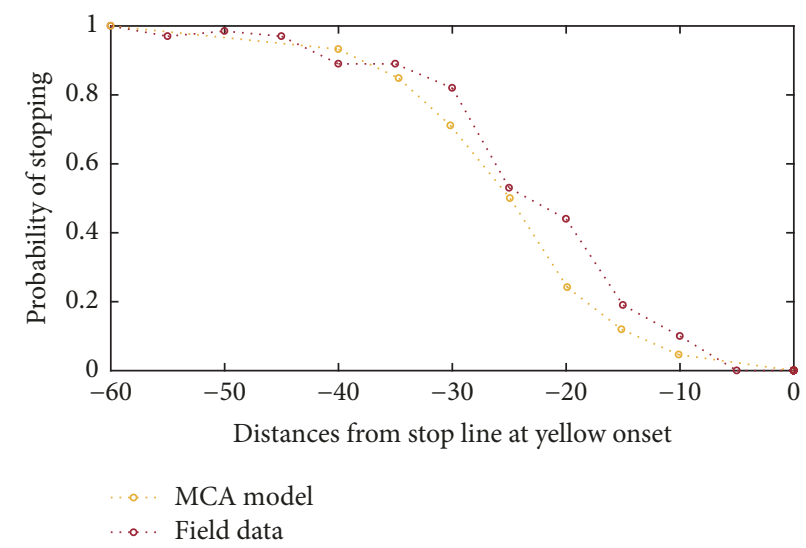

FIGURE 10: Comparison of simulated stopping probability curve and a field curve.

including the distance of the vehicle from the intersection, travel speed at yellow onset, and crossing/stopping decisions. The stopping probabilities at different locations are calculated accordingly. Figure 10 compares the stopping probability curve of the MCA model with that of real data. As shown in Figure 10, the difference between two curves at most distances is within $5 \%$, except for two locations, $20 \mathrm{~m}$ and $30 \mathrm{~m}$, which have a bit higher difference ranging from $10 \%$ to $20 \%$, implying the MCA model can effectively replicate real driver stopping or crossing decision during signal change intervals.

\section{Conclusions}

This study extends the concepts discussed in earlier works $[7,16]$ by further incorporating driver behaviors during change intervals. A modified CA model based on Lan's model for accounting for traffic behaviors, known as perception reaction in response to amber light onset, the decision of a driver to stop/cross, and a reasonable slowdown process are considered.

Compared to Lan et al.s model [7], the exemplified case simulations of vehicular trajectories and deceleration rate show reasonable and practicable slowdown process. According to the macroscopic analysis, only a slight decrease in the maximum flow can be found due to an increase in the amber light duration. However, compared with the entire green-light interval without a change interval, the maximum flow rate is decreased by approximately $18 \%$.

The microscopic analysis reveals that the vehicles passing through the intersection tend to be on the right-upper corner of velocity and space coordinate, suggesting the vehicles with higher speed and closer to the intersection have higher crossing probability while those vehicles stopping at the intersection are of lower speed and locate farer to the intersection. Additionally, the $\mathrm{CB}-\mathrm{SB}$ zone that drivers choose to cross or to stop overlaps widely, implying a lengthy dilemma zone that drivers will encounter. The proposed model can also be used to analyze the effects of driving speed on the dilemma zone which is helpful to better understand the characteristics of traffic flow during the change intervals. The comparisons of the simulated stopping probability curve and field curve show the MCA model can effectively replicate real driver decision in change intervals.

Several directions for future studies can be identified. Firstly, the parameters in this study can be further fine-tuned 
and validated by field traffic data. Secondly, the effect of various offsets on the speed variation of vehicles traversing through two closely consecutive and synchronized intersections can be further explored by the proposed model. Last but not least, it is interesting and essential to further extend the proposed model to replicate mixed traffic behaviors containing cars, trucks, buses, and motorcycles.

\section{Notations}

S: $\quad$ Spatiotemporal domain

$q(S): \quad$ Generalized traffic flow over $S$

$\rho(S)$ : Generalized occupancy over $S$

$|S|: \quad$ Volume of $S$

$N_{0}(t)$ : Total number of sites occupied by cells (vehicles) at the instantaneous time $t$

$N$ : $\quad$ Total number of sites arranged in domain $S$

$t(S)$ : Accumulated $N_{0}(t)$ for all simulated times

$M_{0}(x)$ : Total number of squared sites occupied by cells (representing vehicles) at a specific location $x$ on the road

$d(S)$ : Total distance traveled by all cells in $S$

$\mathrm{Sub}_{n}$ : Subject vehicle's identifier, representing $n$th vehicle within the activation region or not

$P: \quad$ Probability

$P_{b}$ : Impact of decelerating vehicle in near front

$P_{d}: \quad$ Delay-to-start behaviors of vehicles stuck in a traffic jam

$P_{0}: \quad$ Other situations

$P_{a}$ : $\quad$ Reflect uncertainty of driver's decision when encountering amber light

$t$ : Time

$t_{h}: \quad t_{h}=d_{n} / v_{n}(t)$. Time headway of $n$th vehicle to front

$h_{k}$ : $\quad$ Preset time threshold of vehicle of type $k$ for reflecting effect of synchronized distance

$t_{s}: \quad t_{s}=\min \left(v_{n}(t), h_{k}\right)$. Final time threshold for initiating the consideration of front brake light effect, taking vehicular speed into consideration

$t_{\mathrm{st}}: \quad$ Accumulated time of vehicle stuck in traffic jam

$t_{k, c}$ : Time threshold of vehicle of type $k$ for initiating the delay-to-start behavior

$\tau_{n}: \quad$ Safe time gap of $n$th vehicle for collision prevention

$\Delta t: \quad$ Duration of an individual time step

$i$ : $\quad i$ th time step of change intervals duration

$\tau^{a}: \quad$ Amber light duration

$\tau^{r}: \quad$ Red-light duration

$d$ : Space headway

$d_{n}{ }^{\text {eff }}$ : Effective distance of $n$th vehicle

$d_{n}{ }^{\text {sig: }}$ Distance to signal of $n$th vehicle

$g: \quad$ Distance (Gap) $x\left(x^{\mathrm{sig}}\right)$ : Position of $n$th vehicle (position of signal)

$\Delta x: \quad$ Basic unit of roadway length

$d^{\text {act }}:$ Default activation region

$d^{*}: \quad$ Criterion braking distance that corresponds to criterion speed $c^{*}$

$d^{u}$ : $\quad$ Criterion braking distance $d^{*}$ at the last time step

v: $\quad$ Speed

$c n: \quad$ Safe speed of $n$th vehicle

$c^{*}$ : $\quad$ Criterion speed that vehicle can decelerate to stop at a comfortable (fixed) rate per time step

$c^{u}: \quad$ Criterion speed $c^{*}$ at the last time step

A: $\quad$ Acceleration rate

$D$ : $\quad$ Maximum deceleration capacity

$D_{c}$ : $\quad$ Comfortable deceleration rate

rand(): Randomly generated number

$S_{n}: \quad$ Status identifier of $n$th vehicle,

representing its brake light status

$S^{a}{ }_{n}$ : Status identifier of $n$th vehicle, representing go-through or stop status in the amber light duration

$\Delta: \quad$ Minimum clearance for the follower.

Suffix

$n: \quad n$th vehicle

$n+1$ : Vehicle in front

$k$ : $\quad$ Type of vehicles: $k=0$, motorcycle and $k=1$, light vehicle

max: The maximum value.

Superscript

sig: Signal

$a$ : Amber light.

\section{Conflicts of Interest}

The authors declare that they have no conflicts of interest.

\section{Acknowledgments}

This research was supported by Ministry of Science and Technology, Republic of China (MOST 104-2221-E-261-001).

\section{References}

[1] E. Brockfeld, R. Barlovic, A. Schadschneider, and M. Schreckenberg, "Optimizing traffic lights in a cellular automaton model for city traffic," Physical Review E, vol. 64, no. 5, Article ID 056132, 2001.

[2] C. Gershenson and D. A. Rosenblueth, "Modelling selforganizing traffic lights with elementary cellular automata," Complex Systems, vol. 19, no. 4, pp. 305-322, 2009.

[3] T. Nagatani and N. Sugiyama, "Vehicular traffic flow through a series of signals with cycle time generated by a logistic map," Physica A: Statistical Mechanics and its Applications, vol. 392, no. 4, pp. 851-856, 2013. 
[4] H. McGee, K. Moriarty, K. Eccles, M. Liu, T. Gates, and R. Retting, Guidelines for Timing Yellow and All-Red Intervals at Signalized Intersections, Transportation Research Board, Washington, DC, USA, 2012.

[5] T. J. Gates, D. A. Noyce, L. Laracuente, and E. V. Nordheim, "Analysis of driver behavior in dilemma zones at signalized intersections," Transportation Research Record, vol. 2030, pp. 29-39, 2007.

[6] Y. Zhang, C. Fu, and L. Hu, "Yellow light dilemma zone researches: a review," Journal of Traffic and Transportation Engineering (English Edition), vol. 1, no. 5, pp. 338-352, 2014.

[7] L. W. Lan, Y.-C. Chiou, Z.-S. Lin, and C.-C. Hsu, "A refined cellular automaton model to rectify impractical vehicular movement behavior," Physica A: Statistical Mechanics and its Applications, vol. 388, no. 18, pp. 3917-3930, 2009.

[8] L. W. Lan and C.-W. Chang, "Inhomogeneous cellular automata modeling for mixed traffic with cars and motorcycles," Journal of Advanced Transportation, vol. 39, no. 3, pp. 323-349, 2005.

[9] M. E. Lárraga and L. Alvarez-Icaza, "Cellular automaton model for traffic flow based on safe driving policies and human reactions," Physica A: Statistical Mechanics and its Applications, vol. 389, pp. 5425-5438, 2010.

[10] J. P. L. Neto, M. L. Lyra, and C. R. da Silva, "Phase coexistence induced by a defensive reaction in a cellular automaton traffic flow model," Physica A: Statistical Mechanics and its Applications, vol. 390, no. 20, pp. 3558-3565, 2011.

[11] Z. S. Lin, C. C. Hsu, Y. C. Chiou, and L. W. Lan, "Exploring traffic patterns and phase transitions with cellular automaton," Asian Transport Studies, vol. 2, no. 4, pp. 395-410, 2013.

[12] R. Jiang and Q.-S. Wu, "Cellular automata models for synchronized traffic flow," Journal of Physics A: Mathematical and General, vol. 36, no. 2, pp. 381-390, 2003.

[13] W. Knospe, L. Santen, A. Schadschneider, and M. Schreckenberg, "Towards a realistic microscopic description of highway traffic," Journal of Physics A: Mathematical and General, vol. 33, no. 48, pp. 477-485, 2000.

[14] A. Pande and B. Wolshon, Traffic Engineering Handbook, Institute of Transportation Engineers, Washington, DC, USA, 2009.

[15] K. Nagel and M. Schreckenberg, "A cellular automaton model for freeway traffic," Journal de Physique I, vol. 2, no. 12, pp. 22212229, 1992.

[16] L. W. Lan, Y.-C. Chiou, Z.-S. Lin, and C.-C. Hsu, "Cellular automaton simulations for mixed traffic with erratic motorcycles' behaviours," Physica A: Statistical Mechanics and its Applications, vol. 389, no. 10, pp. 2077-2089, 2010.

[17] S. K. Hsu and L. W. Lan, "A comparison of amber-light durations under various approach speeds - Evidence in Taiwan," Journal of the Chinese Institute of Civil and Hydraulic Engineering, vol. 16, no. 3, pp. 543-551, 2004 (Chinese).

[18] C. C. Hsu, Z. H. Lin, Y. C. Chiou, and L. W. Lan, "Exploring traffic features with stationary and moving bottlenecks using refined cellular automata," Journal of the Eastern Asia Society for Transportation Studies, vol. 7, pp. 2502-2516, 2007.

[19] Investigating and Analyzing the Driving Behaviors in Change Intervals at Signalized Intersections, Research Report, Institute of Transportation, Ministry of Transportation and Communications, Taipei, Taiwan, 2002. 


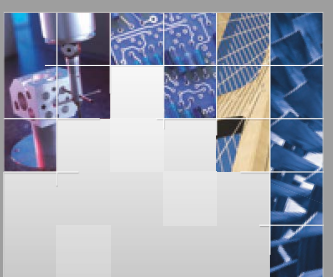

\section{Enfincering}
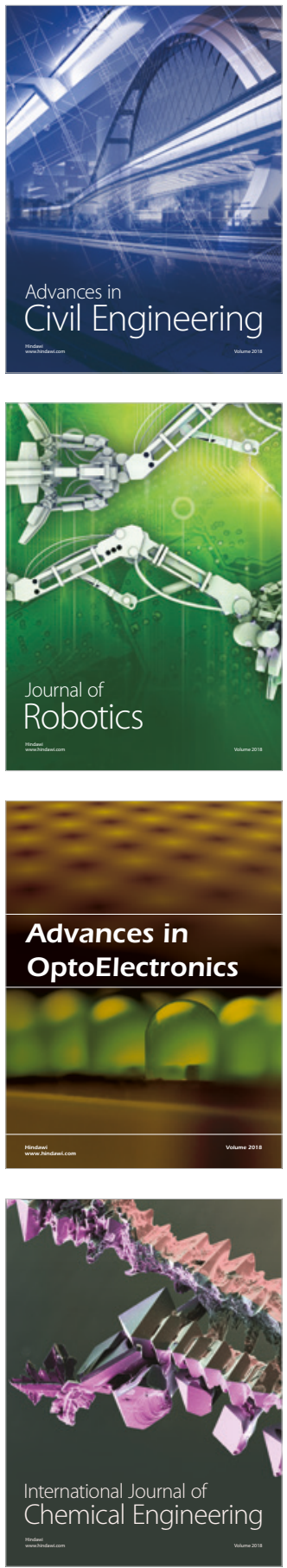

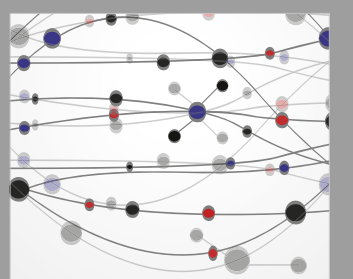

\section{Rotating \\ Machinery}

The Scientific World Journal

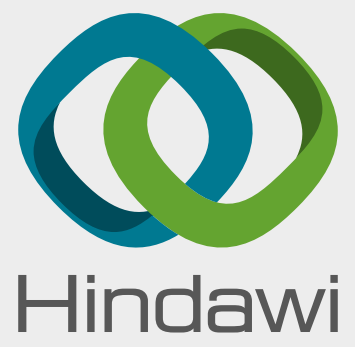

Submit your manuscripts at

www.hindawi.com
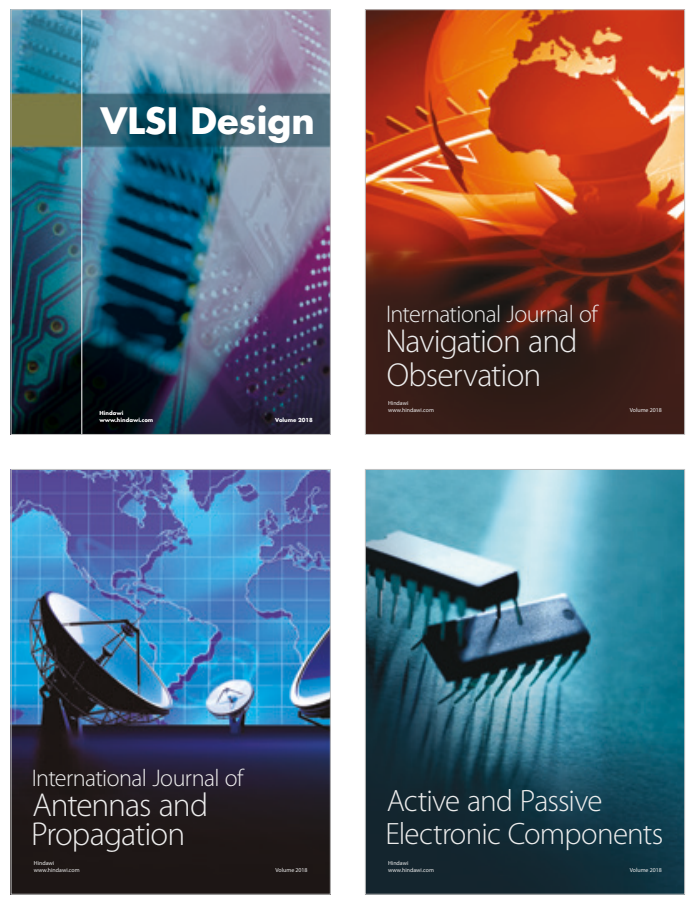
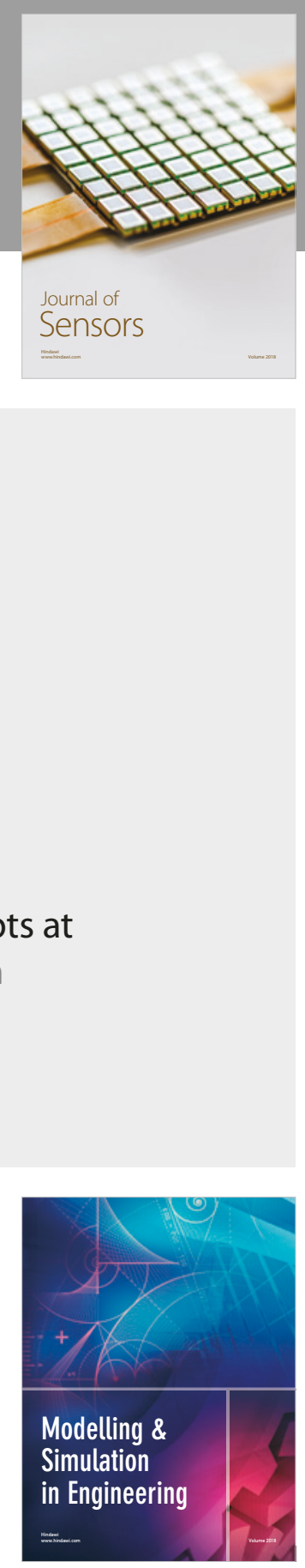

\section{Advances \\ Multimedia}
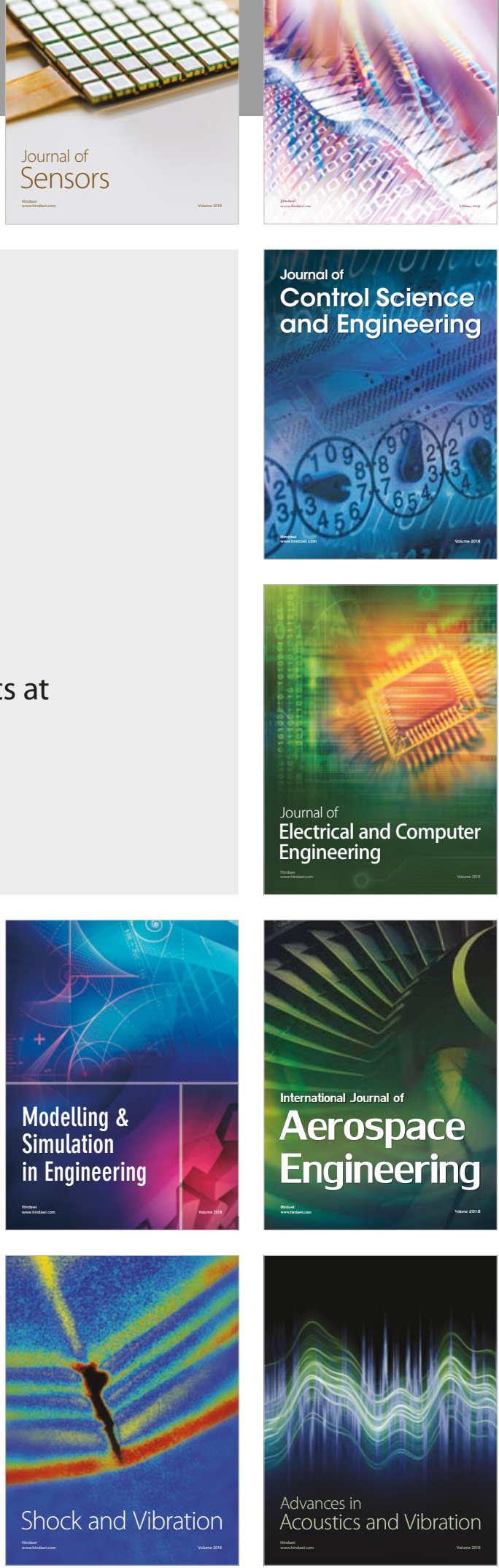